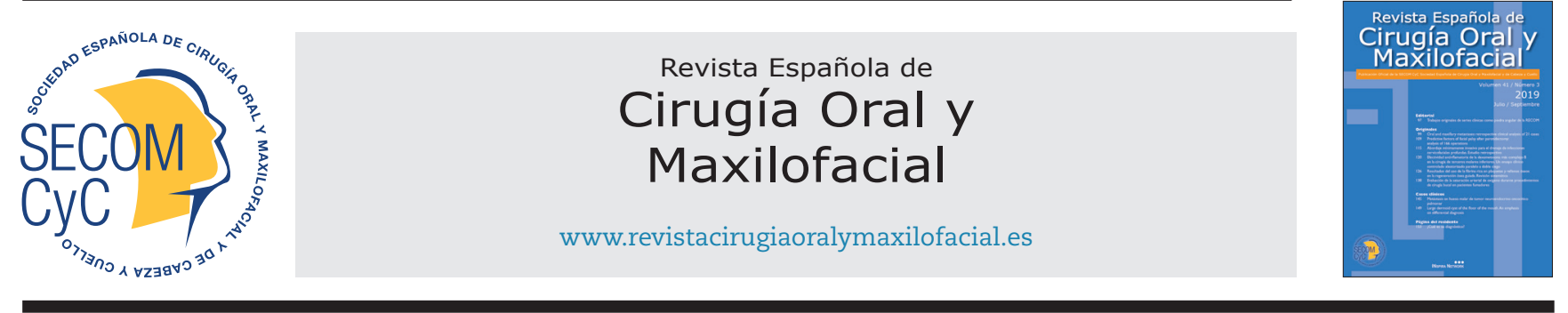

\title{
Original
}

\section{Resultados del uso de la fibrina rica en plaquetas y rellenos óseos en la regeneración ósea guiada. Revisión sistemática}

\author{
Miguel Ángel Núñez Muñoz ${ }^{a}$ y Yuri Castro-Rodríguez ${ }^{b}$
}

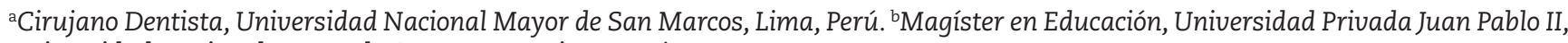
Universidad Nacional Mayor de San Marcos, Lima, Perú

\section{INFORMACIÓN DEL ARTÍCULO}

Historia del artículo:

Recibido el 1 de octubre de 2017

Aceptado el 13 de junio de 2018

\section{Palabras clave:}

Plasma rico en plaquetas, fibrina, regeneración ósea, pérdida de hueso alveolar, seno maxilar, aumento de la cresta alveolar, trasplante óseo.

\section{R E S U M E N}

\begin{abstract}
Objetivo: El propósito de esta revisión fue evaluar sistemáticamente la literatura científica sobre los resultados que se obtienen al combinar la fibrina rica en plaquetas (PRF) y rellenos óseos en la regeneración ósea guiada.

Materiales y métodos: Búsqueda detallada en las bases de datos PubMed, Scopus, Web of Science, ScienceDirect, Cochrane y SciELO para obtener la información más actualizada de los resultados (grado de relleno óseo, éxito de la cirugía, movilidad del implante, complicaciones posquirúrgicas, supervivencia del implante) entre los casos tratados con PRF y los casos donde se combinó PRF con algún tipo de relleno óseo.

Resultados: De las 965 publicaciones identificadas inicialmente, se excluyeron reportes clínicos, revisiones, estudios observacionales, etc. Se incluyeron 12 ensayos clínicos que contrastaron las variables entre la técnica con PRF solo y la combinación con un relleno óseo. Conclusión: La combinación entre PRF más rellenos óseos promueve la neoformación ósea, aumenta el trabeculado y mejora los tiempos de cicatrización; sin embargo, al cabo de 6 meses de control los resultados no son diferentes significativamente de los de los grupos que no utilizaron PRF en el procedimiento de levantamiento de piso de seno maxilar con técnica de ventana lateral. Respecto a la preservación de reborde alveolar, los distintos estudios no son concluyentes: algunos indican que la mezcla de PRF con un relleno óseo parece mejorar las proporciones volumétricas; sin embargo, otros refirieron pérdidas óseas en anchura e incluso mayor grado de inflamación.
\end{abstract}

*Autor para correspondencia:

Correo electrónico: miguelnm8887@gmail.com (Miguel Ángel Núñez Muñoz).

DOI: 10.20986/recom.2019.1032/2019

1130-0558/@ 2019 SECOM. Publicado por Inspira Network. Este es un artículo Open Access bajo la licencia CC BY-NC-ND (http:// creativecommons.org/licenses/by-nc-nd/4.0/). 


\section{Results of platelet rich fibrin and bone grafts in guided bone regeneration. A systematic review}

Keywords:

Plasma rich in platelets, fibrin, bone regeneration, loss of alveolar bone, maxillary sinus, alveolar ridge augmentation, bone transplantation.

\section{A B S T R A C T}

Objective: To evaluate systematically the results obtained by combining platelet rich fibrin (PRF) and bone fill in guided bone regeneration, according to scientific literature.

Materials and method: Detailed search of database from PubMed, Scopus, Web of Science, ScienceDirect, Cochrane and SciELO to obtain the most updated information of clinical results (bone filling degree, surgical success, implant mobility, post-surgical complications, and implant survival) of the cases treated with PRF and the cases where the platelet rich fibrin was combined with a bone filler. Those results registered and compared.

Results: From the 965 files initially identified, were excluded clinical reports, reviews, observational studies, comments, studies with pediatric patients and so on. There were included 12 clinical essays where the variables of the technique with PRF and the technique where the PRF was combined with a bone filler were contrasted.

Conclusion: The combination of PRF plus bone filler promotes bone neoformation, increased trabecular bones, and improved healing times; however, after 6 months of monitoring, the results were not significantly different with the groups that did not use the PRF in the procedure of maxillary sinus floor lift with lateral window technique. Regarding the preservation of alveolar ridge the different studies are not conclusive: some indicate that the mixture of a concentrate with a bone filling seems to improve the volumetric proportions; however, other studies report bone loss in width and even greater degree of inflammation.

\section{INTRODUCCIÓN}

La fibrina rica en plaquetas (PRF) es un concentrado plaquetario de segunda generación que ofrece una elevada cantidad de factores de crecimiento, leucocitos y citocinas que se obtienen mediante la centrifugación de sangre autógena ${ }^{1}$.

Según la clasificación de Dohan-Ehrenfest y cols. ${ }^{2}$, la fibrina rica en plaquetas y leucocitos (L-PRF) se puede obtener mediante un procedimiento muy sencillo y a un bajo coste económico, además de permitir la liberación de una elevada cantidad de factores de crecimiento y proteínas de adhesión ${ }^{3}$. Para la presente revisión el término L-PRF y PRF se utilizan de forma indistinta. El coágulo de L-PRF contiene un $97 \%$ de plaquetas y más del $50 \%$ de los leucocitos del coágulo inicial, y da lugar a una matriz de fibrina capaz de liberar factores de crecimiento y proteínas implicadas en la curación de heridas durante más de 7 días in vitro ${ }^{3-9}$.

Se ha demostrado que el uso del L-PRF en combinación con sustitutos óseos presenta un excelente comportamiento como conector biológico entre las partículas óseas,30-12. La combinación parece tener un efecto sinérgico en el proceso de cicatrización y regeneración; sin embargo, se ha observado que sus efectos no son duraderos a largo plazo debido a la rápida acción de sus proteínas bioactivas ${ }^{13}$.

Actualmente se están intentando utilizar diferentes activadores $^{14}$ y transportadores ${ }^{15}$ para prolongar el efecto del PRF. Las mezclas parecen acelerar la regeneración ósea y permiten colocar los implantes dentales en menos tiempo ${ }^{16}$. Es difícil determinar si la adición del PRF permite mejorar el depósito de nuevo hueso y, por ende, acelerar el proceso de regeneración ósea. Surge así la necesidad de conocer si los ensayos clínicos realizados en los procedimientos de regeneración ósea guiada (ROG) encuentran resultados eficaces. La presente revisión sistemática sinteti- zó las publicaciones más actualizadas sobre los resultados de la combinación de PRF más rellenos óseos en las técnicas de ROG.

\section{MATERIALES Y MÉTODOS}

\section{Protocolo PICO}

La revisión sistemática siguió el siguiente protocolo: Población (P): pacientes sistémicamente sanos (ASA I) con atrofia de reborde alveolar con/sin la necesidad de colocación de implantes dentales. Intervención (I): aplicación del PRF como biomaterial (solo o en combinación con rellenos óseos) en la ROG. Comparación (C): técnicas clásicas que utilizan rellenos óseos (aloinjertos, xenoinjertos, aloplásticos) con o sin el uso de membranas de colágeno o injertos conectivos subepiteliales. Resultado (O): regeneración ósea alveolar (expresado como porcentaje de formación ósea, cicatrización de tejido óseo y de tejidos blandos, grado de reabsorción ósea) y oseointegración implantaria (expresada como valores de inserción del torque del implante y la pérdida ósea marginal en milímetros).

\section{Estrategia de búsqueda}

Los autores exploraron los artículos en las bases de datos PubMed, Scopus, Web of Science, ScienceDirect, Cochrane y SciELO desde enero de 2009 hasta julio del 2017. La limitación temporal tenía como fin la búsqueda de los artículos más actuales y que utilizasen las técnicas más comunes. La investigación fue complementada con una búsqueda manual en el mismo periodo en las revistas: Journal of Periodontology, International Journal of Oral \& Maxillofacial Implants, International Journal 
of Oral \& Maxillofacial Surgery, Journal of Oral and Maxillofacial Surgery, Journal of Dental Research, Journal of Clinical Periodontology, International Journal of Periodontics \& Restorative Dentistry y European Journal of Oral Implantology. Una vez encontrados los artículos, se complementó con una búsqueda a través de las referencias bibliográficas para detectar aquellas publicaciones que no fueron identificadas electrónicamente.

\section{Términos de búsqueda}

Las palabras clave seleccionadas fueron: ["Guided Bone Regeneration"-MeSH-] o ["Bone" y "regeneration" -todos los campos-] o ["Platelet-Rich Fibrin"-MeSH-] o ["Platelet-Rich Plasma" y "PlateletRich-Fibrin" -todos los campos-] o ["Dental Implants" -Todos los campos-] o ["Surgery techniques" -MeSH-] o ["Clinical Trial" -todos los campos-].

\section{Criterios de inclusión y exclusión}

Se incluyeron: publicaciones en inglés o español; ensayos clínicos que comparasen los resultados entre PRF versus PRF más rellenos óseos en la ROG; ensayos clínicos que comparasen los resultados entre PRF versus PRF más membranas de colágeno/injertos conectivos en la ROG; ensayos clínicos que evaluaran las características de grado de relleno óseo, complicaciones posquirúrgicas, éxito de la cirugía y supervivencia del implante. Se excluyeron: estudios preclínicos o estudios piloto; ensayos clínicos que no describiesen los resultados postoperatorios de grado de relleno óseo, complicaciones posquirúrgicas, éxito de la cirugía y supervivencia del implante; estudios clínicos de los tipos: reportes de caso, series de caso, estudios retrospectivos y revisiones sistemáticas.

\section{Proceso de selección}

Inicialmente fueron seleccionados todos los títulos, para después eliminar las publicaciones no relevantes; en la primera búsqueda se encontraron 965 artículos. Durante una segunda etapa se utilizaron los filtros de cada base de datos seleccionando las opciones de "búsqueda por tiempo", "búsqueda de ensayos clínicos" y "búsqueda de artículos", principalmente en las bases Scopus, Web of Science, PubMed y ScienceDirect. Los artículos resultantes fueron analizados a través de sus resúmenes; los dos autores (MN y YC) seleccionaron de forma independiente todos los resúmenes. El grado de concordancia kappa entre los dos autores fue de 0,89 para la concordancia de selección de los resúmenes. Luego se obtuvieron los artículos que cumplían los criterios de inclusión. Tras esta búsqueda, se analizaron los artículos relevantes y las referencias bibliográficas para añadir más artículos que cumplían con los criterios (Figura 1).

\section{Valoración de la calidad científica de los estudios}

Para la evaluación de la calidad metodológica, los datos de cada estudio fueron analizados según la escala de Jadad ${ }^{17} \mathrm{y}$ la escala de Downs y Black ${ }^{17}$ (Check list for Measuring Quality o CMQ) modificada con 27 ítems, eliminando las preguntas relacionadas con estudios experimentales $(4,8,12,13,24$ y 27$)$. Si algún estudio obtenía en la escala de Jadad un valor menor a 3, el artículo era evaluado mediante la escala CMQ para poder determinar si se incluía en el estudio (Tabla I).

\section{Extracción de datos}

Los datos extraídos incluyeron: 1) técnica utilizada/tipo de relleno óseo; 2) diseño del estudio y tiempo de seguimiento; 3) tamaño de la muestra; 4) características individuales y hábitos de los pacientes, y 5) evaluación de la neoformación ósea.

\section{RESULTADOS}

La selección de los 438 artículos filtrados excluyó 381 según sus títulos y resúmenes, resultando así 57 artículos potencialmente relevantes. La lectura completa de cada artículo excluyó 45, quedando así 12 artículos seleccionados para el análisis y extracción de datos.

Ocho artículos ${ }^{11,12,16,18-22}$ se relacionaron con la atrofia del maxilar e indicación de levantamiento de piso del seno maxilar con la técnica de Boyne y James ${ }^{23}$ y Tatum ${ }^{24}$. Algunos estudios $^{11,16,18-22}$ evaluaron el efecto del PRF en los procedimientos de ROG tratando en total 188 senos maxilares. Sus análisis histomorfométrico ${ }^{20-22}$ indicaron que, al combinar hueso bovino con PRF, el porcentaje de formación ósea osciló entre el 18,35 \% y el $35 \%$, mientras que al combinar aloinjerto óseo liofilizado con PRF, el porcentaje de formación ósea fue del $20,95 \%^{16}$, sin obtener diferencias estadísticamente significativas. La combinación de $\beta$-fosfato tricálcico ( $\beta$-TCP) con PRF arrojó un porcentaje de formación ósea del 32,03\% ${ }^{18}$. Al combinar hueso autólogo con hueso bovino y con PRF, el porcentaje de hueso vital fue del $17 \%{ }^{19}$. En un estudio ${ }^{12}$, al combinar $\beta$-TCP con A-PRF, se encontraron mayores valores de torque de inserción del implante que los implantes que no utilizaron la combinación $(p<0,05)$ (Tabla II).

Dos artículos ${ }^{25,26}$ estuvieron relacionados con el tratamiento de las hendiduras alveolares maxilares unilaterales. Uno de los estudios $^{25}$ comparó el uso del injerto óseo iliaco versus hueso de mentón más relleno alogénico más PRF, obteniéndose una ganancia ósea de volumen del 69,57 \% en el grupo PRF. En el otro estudio ${ }^{26}$ se utilizó el injerto óseo iliaco versus injerto óseo iliaco más PRF, obteniéndose una ganancia ósea del 82,61 \% en el grupo de PRF.

Dos artículos evaluaron la preservación de rebordes alveolares $^{27,28}$. Uno comparó dos grupos ${ }^{27}$ : exodoncia más PRF versus exodoncia con colgajo más PRF; el grupo sin colgajo presentó un porcentaje significativo $(p<0,05)$ menor de pérdida de anchura alveolar (-0,48 \%). El otro estudio ${ }^{28}$ comparó la exodoncia versus exodoncia más PRF, evidenciándose una pérdida ósea marginal para el grupo control de 2,48 $\mathrm{mm}$ y de $2,15 \mathrm{~mm}$ para el grupo experimental, sin diferencias significativas $(p>0,05)$.

\section{DISCUSIÓN}

Los estudios relacionados con la elevación de piso de seno maxilar mediante la técnica de ventana lateral refirieron que al cabo de 6 meses se encuentra mayor neoformación ósea al 


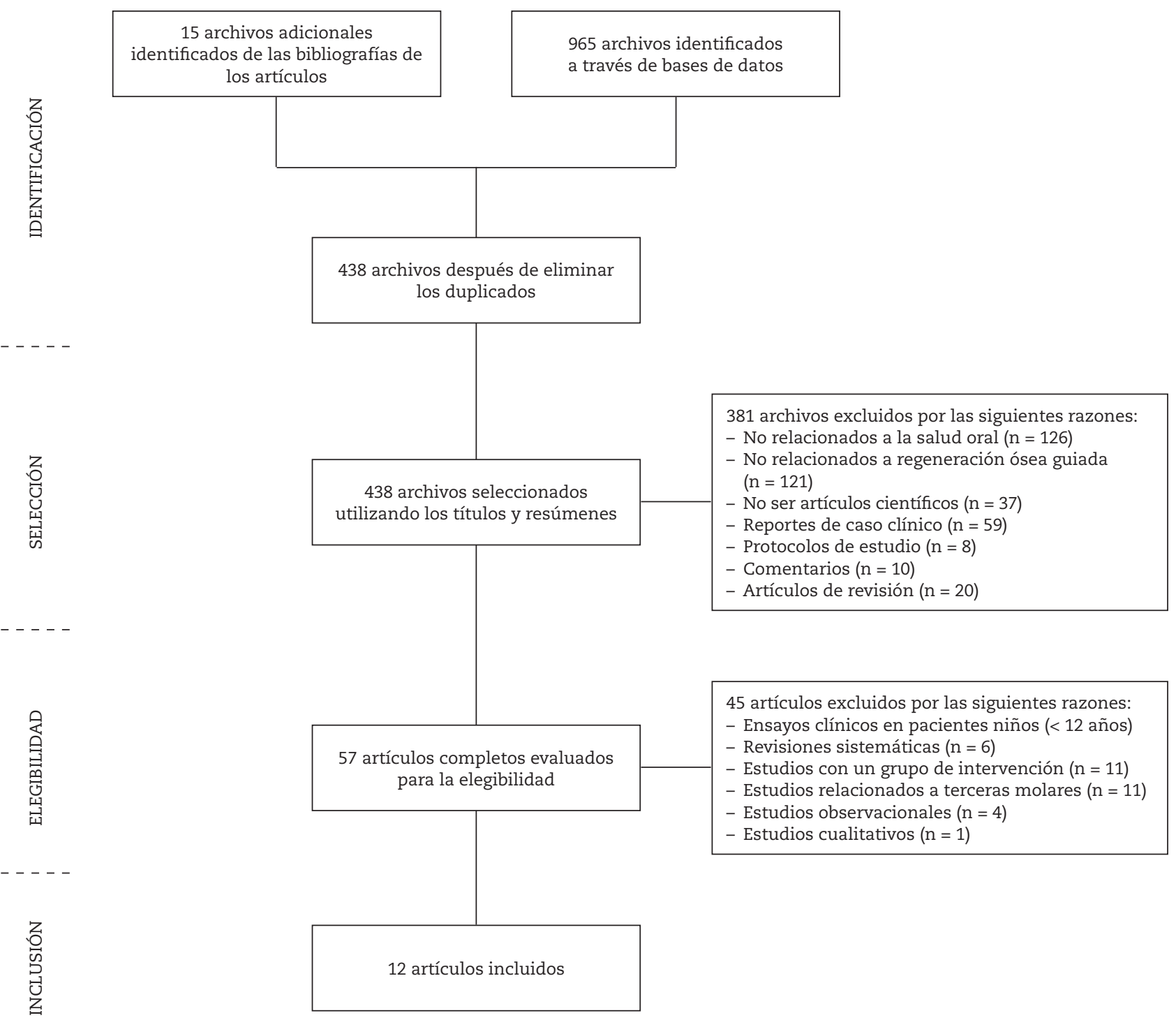

Figura 1. Diagrama de flujo de la revisión sistemática. Se incluyeron 12 publicaciones en el análisis.

combinar plasma rico en plaquetas (PRP) o PRF con $\beta$-TCP ${ }^{29-33}$. Se conoce que los concentrados otorgan los factores de crecimiento al $\beta$-TCP. De esta forma la neoformación ósea aumentaría, aunque algunos estudios solo han encontrado entre el 19 y el $30 \%$ de formación ósea ${ }^{34-37}$; quizás porque no hay beneficio en el aumento de células, vasos capilares y nueva formación ósea, además de que los resultados encontrados a largo plazo no evidenciaron diferencias significativas con los grupos que utilizaron la mezcla PRF y $\beta$-TCP ${ }^{18,38,39}$.

La liberación de factores de crecimiento estimula las células cercanas a la quimiotaxis ${ }^{40}$, proliferación y maduración ${ }^{41,42}$; sin embargo, los rellenos óseos como el $\beta$-TCP carecen de células óseas libres, por lo que el potencial efecto del PRP o PRF sería nulo ${ }^{43,44}$. Esto podría explicar la no superioridad de la mezcla, además que la lenta degradación del $\beta$-TCP puede retardar la capacidad de ROG del PRP o PRF $21,45,46$.

Respecto al hueso medular desmineralizado bovino, se conoce que es osteoconductivo y permite el transporte de células osteogenéticas desde la pared del seno maxilar hasta las partículas del injerto ${ }^{47-49}$. Su uso en forma de Bio-Oss (hueso bovino desproteinizado) ha demostrado mayor neoformación ósea al mezclarlo con $\mathrm{PRF}^{21,22}$. También se ha descrito que las partículas de Bio-Oss se incorporan en el hueso recién formado y la proporción de hueso neoformado aumenta con un mayor periodo de observación ${ }^{50,51}$. Quizás se deba al largo tiempo de reabsorción del hueso bovino $0^{50,52-55}$. Sin embargo, los estudios de largo plazo que evaluaron la biodegradación de Bio-Oss son escasos; los informes de casos han demostrado la presencia de partículas de Bio-Oss 9-10 años después de elevaciones de pisos de seno maxilar ${ }^{56,57}$.

Se ha sugerido que el PRF es una membrana bioactiva para la ROG, pero solo se han publicado informes clínicos sobre este tema ${ }^{58-61}$. Debido a la preocupación por la degradación de la membrana de PRF, recientemente Kawase y cols. ${ }^{62}$ lograron reducir la tasa de biodegradación de la membrana de PRF utilizando una técnica de compresión por calor que no involucraba su biocompatibilidad. 
Jadad

¿Se describe el estudio como aleatorizado?

¿Se describe el método utilizado para generar la secuencia de aleatorización y este método es adecuado?

¿Se describe el estudio como doble ciego?

¿Se describen las pérdidas y retiradas del estudio?

¿Es adecuado el método de enmascaramiento?

Total

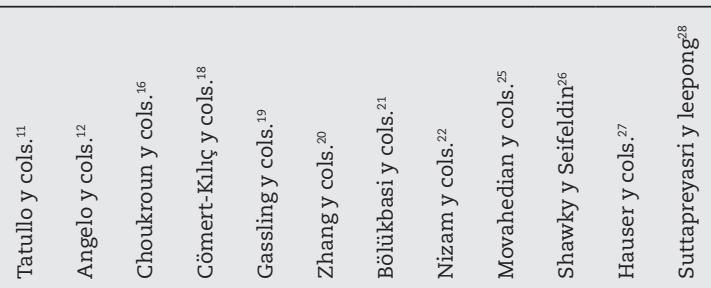

¿Se describe claramente la hipótesis/objetivo del estudio?
¿Se describen claramente los criterios de valoración principales en el
apartado de introducción o métodos?
¿Se describen claramente las características de los pacientes incluidos
estudio?
¿Se describen claramente las distribuciones de los principales factores
confusión en cada grupo de sujetos en comparación?
¿Se describen claramente los resultados principales del estudio?
¿Proporciona el estudio estimaciones de la variabilidad aleatoria en los
datos relativos a los criterios de valoración principales?
¿Se han descrito las características de los pacientes perdidos para el
seguimiento?

¿Se han comunicado los valores de probabilidad reales (por ejemplo, 0,035 en lugar de $<0,05$ ) para los criterios de valoración principales salvo cuando los valores de probabilidad son inferiores a 0,001?

¿Eran los sujetos a los que se pidió participar en el estudio representativos de toda la población a partir de la cual fueron seleccionados?

¿Los sujetos de estudio fueron ciegos ante la intervención que recibieron? ¿Se cegó a las personas que midieron los principales resultados de la intervención?

En caso de que alguno de los resultados del estudio se basara en un

"dragado de datos", ¿se indicó claramente?

En los ensayos y estudios de cohortes, ¿se ajustaron los análisis en función de diferentes duraciones del seguimiento de los pacientes?, o en los estudios de casos y controles, ¿fue el tiempo transcurrido entre la intervención y el criterio de valoración el mismo para los casos y los controles?

$\begin{array}{llllllllllll}1 & 1 & 1 & 1 & 1 & 1 & 0 & 1 & 1 & 1 & 1 & 1 \\ 1 & 1 & 1 & 1 & 1 & 1 & 0 & 1 & 1 & 1 & 1 & 0\end{array}$
1 


\begin{tabular}{|c|c|c|c|c|c|c|c|c|c|c|c|c|}
\hline \multicolumn{13}{|l|}{$\mathrm{CMQ}^{\mathrm{b}}$} \\
\hline $\begin{array}{l}\text { ¿Fueron exactos (válidos y fiables) los criterios de valoración principales } \\
\text { utilizados? }\end{array}$ & 1 & 1 & 1 & 1 & 1 & 1 & 1 & 1 & 1 & 1 & 1 & 1 \\
\hline $\begin{array}{l}\text { ¿Se seleccionó a los pacientes incluidos en diferentes grupos de } \\
\text { intervención (ensayos y estudios de cohorte) o los casos y controles } \\
\text { (estudios de casos y controles) a partir de la misma población? }\end{array}$ & 1 & 1 & 1 & 1 & 1 & 1 & 1 & 1 & 1 & 1 & 1 & 1 \\
\hline $\begin{array}{l}\text { ¿Se seleccionó a los sujetos incluidos en diferentes grupos de intervención } \\
\text { (ensayos y estudios de cohorte) o los casos y controles (estudios de casos y } \\
\text { controles) durante el mismo periodo de tiempo? }\end{array}$ & 1 & 1 & 1 & 1 & 1 & 1 & 1 & 1 & 1 & 1 & 1 & 1 \\
\hline $\begin{array}{l}\text { ¿Los sujetos de estudio fueron asignados al azar a los grupos } \\
\text { deintervención? }\end{array}$ & 1 & 1 & 0 & 1 & 1 & 1 & 1 & 1 & 1 & 1 & 1 & 1 \\
\hline $\begin{array}{l}\text { ¿Hubo un ajuste adecuado respecto a factores de confusión en los análisis a } \\
\text { partir de los que se extrajeron los resultados principales? }\end{array}$ & 0 & 0 & 0 & 0 & 0 & 0 & 0 & 0 & 0 & 0 & 0 & 0 \\
\hline ¿Se tuvieron en cuenta las pérdidas de pacientes para el seguimiento? & 0 & 0 & 0 & 0 & 0 & 0 & 0 & 0 & 0 & 0 & 0 & 0 \\
\hline Total & 19 & 19 & 15 & 18 & 18 & 18 & 16 & 18 & 19 & 18 & 19 & 18 \\
\hline $\begin{array}{l}{ }^{a} \text { Resultados: } 5 / 5 \text { = calidad metodológica excelente; } 4 / 5=\text { calidad metodológica buena; 3/5 } \\
\text { pobre; } 1 / 5=\text { calidad metodológica deficiente. } \\
{ }^{b} \text { Resultados: } 20-22 \text { = calidad metodológica excelente; } 15-19 \text { = calidad metodológica buena } \\
\text { metodológica pobre; } 0-4 \text { = calidad metodológica deficiente. } \\
\text { Análisis de la calidad metodológica de los estudios. Nueve estudios (referencias 11, } 12,16 \\
\text { Jadad superior a 2, lo que los califica como estudios con una calidad metodológica aceptab } \\
\text { 27). Los estudios que obtuvieron una puntuación menor a } 3 \text { según la escala de Jadad (refe } \\
\text { la que obtuvieron unas puntaciones de } 16 \text { (referencia 21) y de } 18 \text { (referencias } 18 \text { y 28), lo } \\
\text { hace viable su inclusión en el presente estudio. } \\
\text { CMQ: Check list for Measuring Quality. }\end{array}$ & $\begin{array}{l}9,2 \\
\text { (ref } \\
\text { ncia }\end{array}$ & $\begin{array}{l}22, \\
\text { enci } \\
18,2\end{array}$ & & $\begin{array}{l}\text { y } 27 \\
16,1 \\
p a\end{array}$ & 20 & $\begin{array}{l}\text { ero } \\
\text { ser }\end{array}$ & & $\begin{array}{l}\text { lor } \\
\text { y } b \\
\text { dos }\end{array}$ & jún & $d$ & 1 la & \\
\hline
\end{tabular}

Respecto al empleo de hueso autólogo en la ROG, se ha utilizado el injerto óseo autógeno de la cresta iliaca como el estándar para estimular la cicatrización ósea y rellenar los defectos óseos grandes ${ }^{63-66}$. Los procedimientos para regenerar hendiduras alveolares maxilares mediante este tipo de injerto demostraron mayor formación ósea cuando se combina con $\mathrm{PRF}^{26}$, mientras que en otro estudio los cambios no fueron diferentes estadísticamente ${ }^{25}$.

La reabsorción ósea alveolar tras de la exodoncia dental es un proceso inevitable. En relación con los rebordes alveolares, se conoce que el reborde alveolar residual se reduce en anchura y altura, siendo mayor en anchura ${ }^{67}$, mayor en la zona bucal ${ }^{68,69}$ y más rápido durante los seis primeros meses ${ }^{70-72}$. Se ha reportado que la realización de una exodoncia combinada con el uso del PRF reduce la reabsorción de las crestas marginales ${ }^{28}$. No obstante, Araujo y Lindhe ${ }^{73}$ indican que la cresta bucal migra constantemente hacia apical después de una exodoncia.

Hauser y cols. ${ }^{27}$ también encontraron menor reducción del ancho de la cresta alveolar en el procedimiento de exodoncia más PRF y la mayor reducción del ancho de la cresta alveolar en los procedimientos de exodoncia más colgajo y PRF. Algunos estudios ${ }^{74-76}$ refieren que utilizar un colgajo mucoso presenta desventajas significativas, ya que la elevación del periostio del hueso comprometería el suministro vascular a la superficie del hueso, lo que conduciría a la actividad osteoclástica y al aumento de la reabsorción ósea.

La presente revisión sistemática posee algunas limitaciones que se han de tener en cuenta: en primer lugar, la mayoría de los estudios analizados presentaron riesgos de sesgos moderados y elevados. La heterogeneidad de estudios y variables analizadas no permitió la realización de un metaanálisis, y principalmente se analizaron resultados desde el punto de vista cualitativo. Se resalta que la mayoría de ensayos clínicos estuvieron relacionados con la técnica de levantamiento de piso de seno maxilar, con una elevada cantidad de pacientes evaluados.

\section{CONCLUSIONES}

La combinación de PRF y rellenos óseos promueve la neoformación ósea, aumenta el trabeculado y reduce los tiempos de cicatrización en los procedimientos de elevación de piso de seno maxilar; sin embargo, al cabo de seis meses de control los resultados no son diferentes significativamente cuando se comparan con los obtenidos por los grupos que no utilizan PRF. 


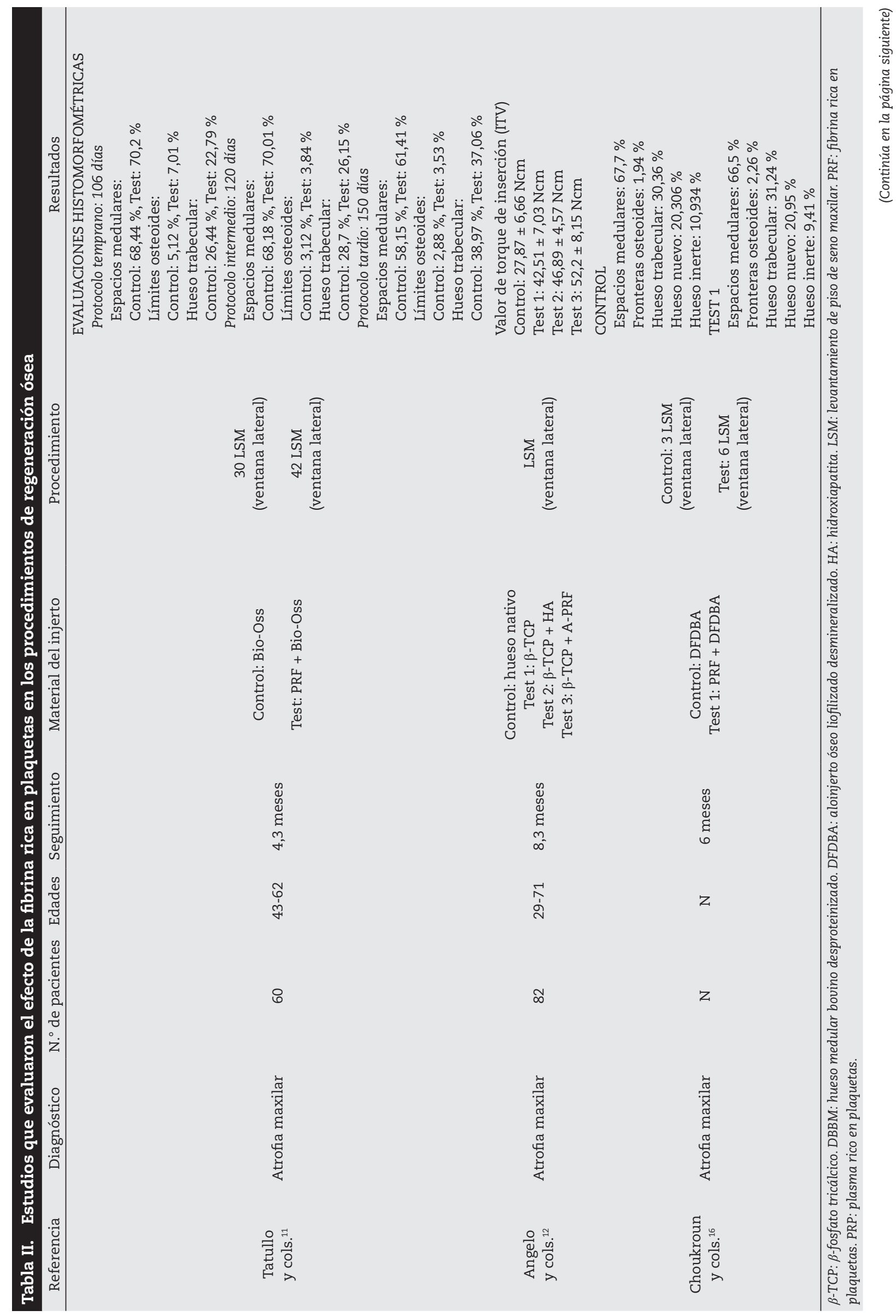




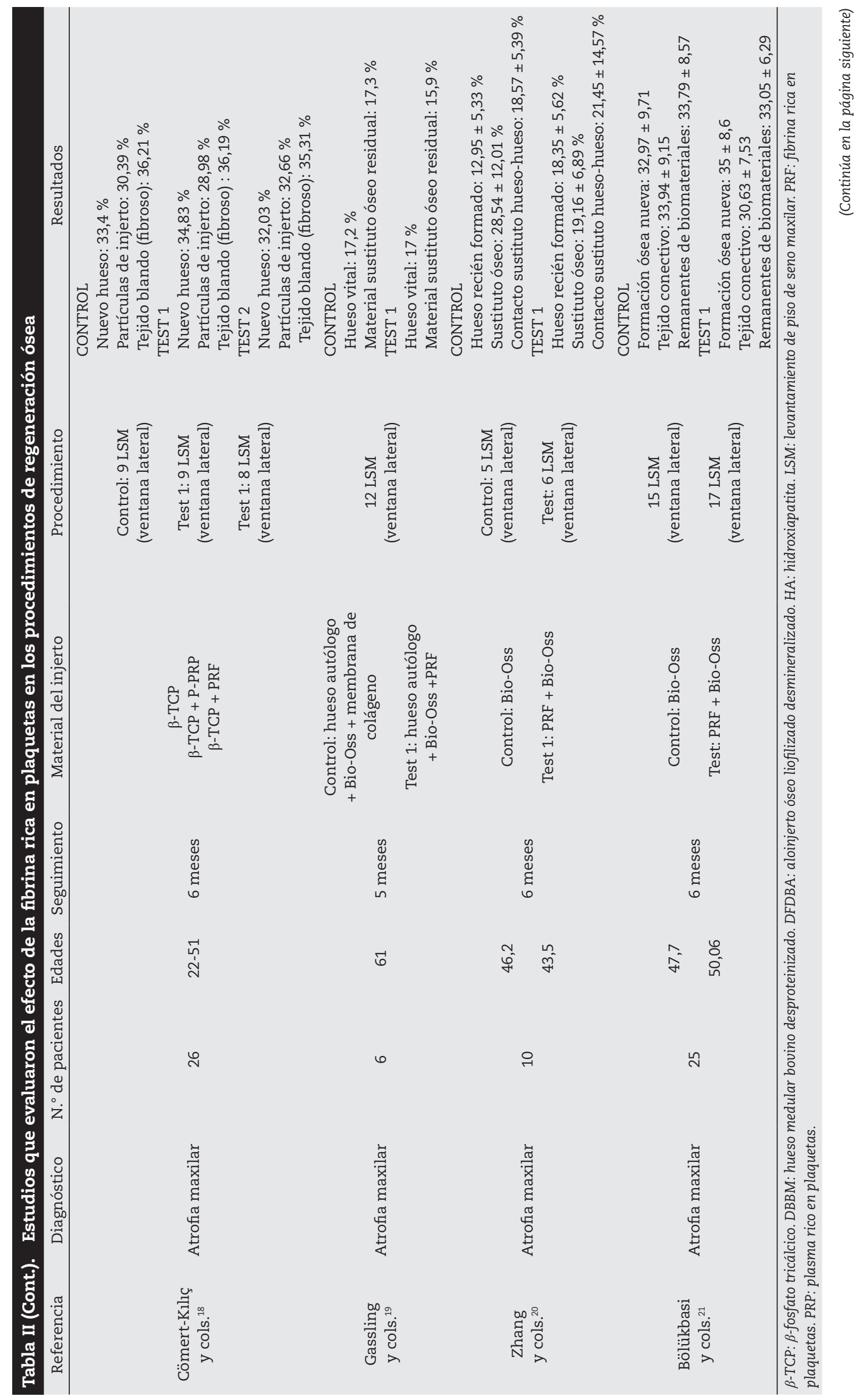



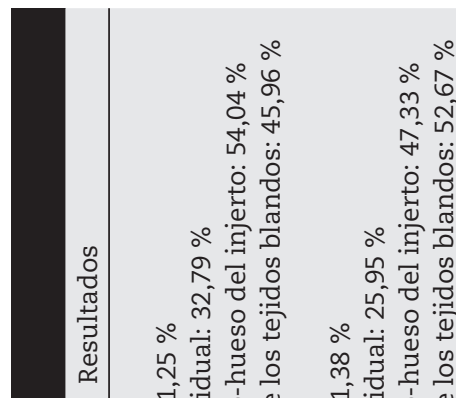

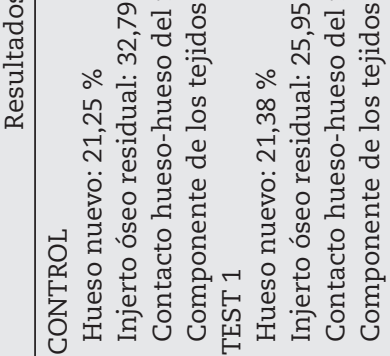

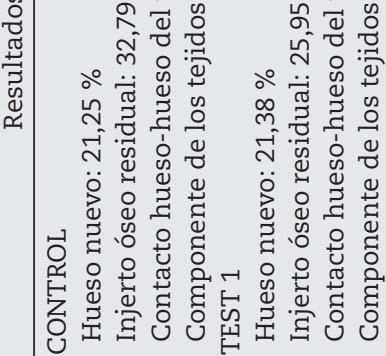

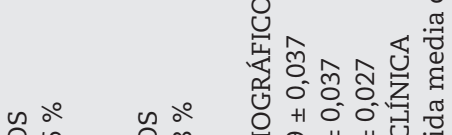

O
剀
0

$\stackrel{\infty}{\infty} \circ$

赵

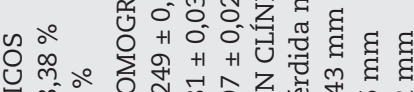

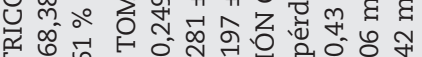

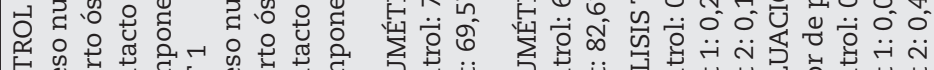

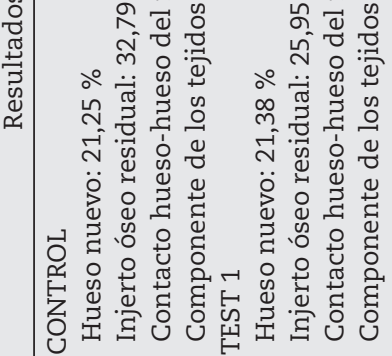

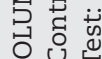

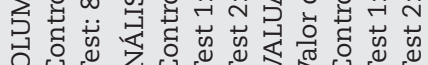
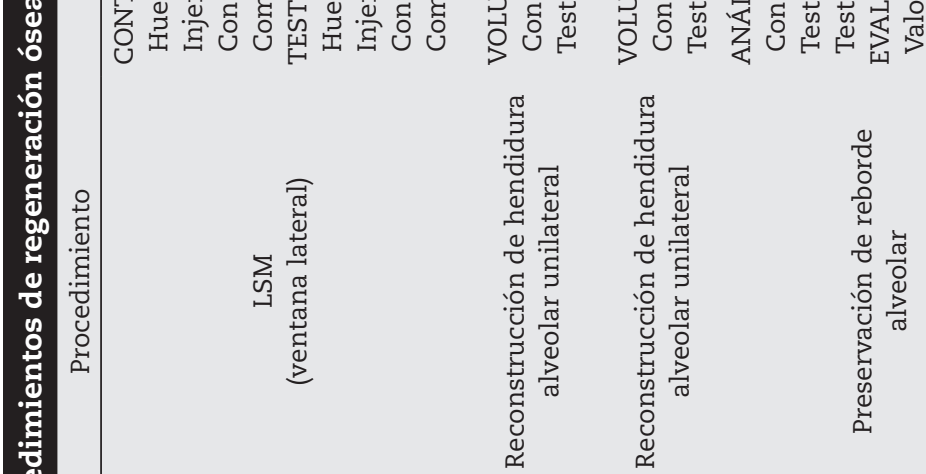

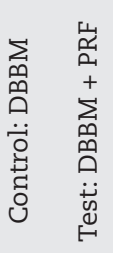
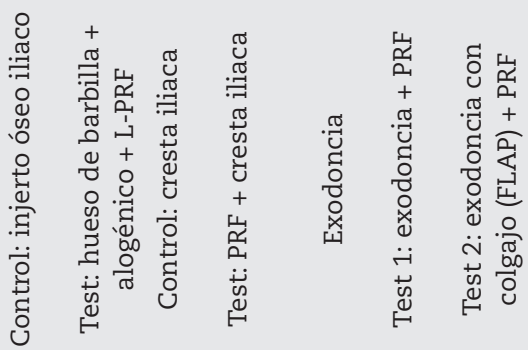

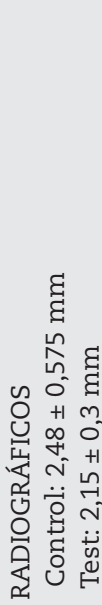

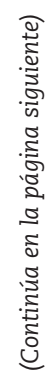

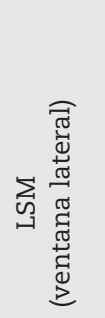

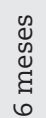

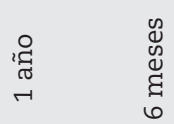

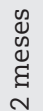

+1
Nू
के
oे

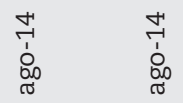

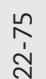
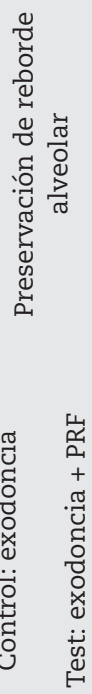
Los resultados de la combinación de PRF y rellenos óseos en preservaciones del reborde alveolar aún no son claros, pero se reporta el beneficio de la matriz de fibrina en el cierre de la herida y la reepitelización.

Los estudios relacionados con el tratamiento regenerativo de las hendiduras alveolares evidencian una notoria ROG al combinar hueso autólogo con PRF.

\section{CONFLICTO DE INTERESES}

Los autores declaran no tener conflictos de interés.

\section{FUENTE DE FINANCIACIÓN}

Autofinanciado.

\section{B I B L I O G R A F Í A}

1. Meza-Mauricio EJ, Lecca-Rojas MP, Correa-Quispilaya E, RíosVillasis K. Fibrina rica en plaquetas y su aplicación en Periodoncia: Revisión de literatura. Rev Estomatol Herediana. 2014;24(4):287-93.

2. Dohan-Ehrenfest DM, Rasmusson L, Albrektsson T. Classification of platelet concentrates: From pure platelet-rich plasma (P-PRP) to leucocyte- and platelet-rich fibrin (L-PRF). Trends Biotechnol. 2009;27(3):158-67. DOI: 10.1016/j.tibtech.2008.11.009.

3. Salgado-Peralvo AO, Salgado-García A, Arriba-Fuente L. Nuevas tendencias en regeneración tisular: fibrina rica en plaquetas y leucocitos. Rev Esp Cirug Oral y Maxilofac. 2017;39(2):91-8. DOI: 10.1016/j.maxilo.2016.03.001.

4. Del Corso M, Vervelle A, Simonpieri A, Jimbo R, Inchingolo F, Sammartino G, et al. Current knowledge and perspectives for the use of platelet-rich plasma (PRP) and platelet-rich fibrin (PRF) in oral and maxillofacial surgery. Part I: Periodontal and dentoalveolar surgery. Curr Pharm Biotechnol. 2012;13(7):1207-30.

5. Mazor Z, Horowitz RA, del Corso M, Prasad HS, Rohrer MD, Dohan-Ehrenfest DM. Sinus floor augmentation with simultaneous implant placement using Choukroun's platelet-rich fibrin as the sole grafting material: a radiologic and histologic study at 6 months. J Periodontol. 2009;80(12):2056-64. DOI: 10.1902/jop.2009.090252.

6. Del Corso M, Toffler M, Dohan-Ehrenfest DM. Use of an autologous leukocyte and platelet-rich fibrin (L-PRF) membrane in post-avulsion sites: An overview of Choukroun's PRF. J Int Am Clin Dent. 2010;1(9):27-35.

7. Khiste SV, Tari RN. Platelet-rich fibrin as a biofuel for tissue regeneration. ISRN Biomaterials. 2013;2013:1-6.

8. Dohan-Ehrenfest DM, Sammartino G, Shibli JA, Wang HL, Zou DR, Bernard JP. Guidelines for the publication of articles related to platelet concentrates (platelet-rich plasma - PRP, or platelet-rich fibrin - PRF): The International Classification of the POSEIDO. Periodontol Oral Surg Esthetic Implant Dent Op. J 2013;1(1):17-27.

9. Del Corso M, Dohan-Ehrenfest DM. Immediate implantationand peri-implant natural bone regeneration (NBR) in theseverely resorbed posterior mandible using leulocyte- and platelet-rich fibrin (L-PRF): A 4-year follow-up. Periodontol Oral Surg Esthetic Implant Dent Op J. 2013;1(2):109-16.

10. Cieslik-Bielecka A, Dohan-Ehrenfest DM, Lubkowska A, Bielecki T. Microbicidial properties of leukocyte- andplatelet-rich plasma/fibrin (L-PRP/L-PRF): New perspectives. J Biol Regul Homeost Agents. 2012;26(2 suppl 1):43S-52S.
11. Tatullo M, Marrelli M, Cassetta M, Pacifici A, Stefanelli LV, Scacco S, et al. Platelet Rich Fibrin (P.R.F.) in reconstructive surgery of atrophied maxillary bones: clinical and histological evaluations. Int J Med Sci. 2012;9(10):872-80. DOI: 10.7150/ijms.5119.

12. Angelo T, Marcel W, Andreas K, Izabela S. Biomechanical stability of dental implants in augmented maxillary sites: Results of a randomized clinical study with four different biomaterials and PRF and a biological view on guided bone regeneration. BioMed Res Int. 2015;2015:850340. DOI: 10.1155/2015/850340.

13. Schmitz JP, Hollinger JO. The biology of platelet-rich plasma. J Oral Maxillofac Surg. 2001;59(9):1119-21. DOI: 10.1053/joms.2001.26801.

14. Anitua E. Plasma rich in growth factors: preliminary results of use in the preparation of future sites for implants. Int J Oral Maxillofac Implants. 1999;14(4):529-35.

15. Hokugo A, Sawada Y, Hokugo R, Iwamura H, Kobuchi M, Kambara $\mathrm{T}$, et al. Controlled release of platelet growth factors enhances bone regeneration at rabbit calvaria. Oral Surg Oral Med Oral Pathol Oral Radiol Endod. 2007;104(1):44-8. DOI: 10.1016/j.tripleo.2006.11.032.

16. Choukroun J, Diss A, Simonpieri A, Girard MO, Schoeffler C, Dohan SL, et al. Platelet-rich fibrin (PRF): a second-generation platelet concentrate. Part V: histologic evaluations of PRF effects on bone allograft maturation in sinus lift. Oral Surg Oral Med Oral Pathol Oral Radiol Endod. 2006;101(3):299-303. DOI: 10.1016/j.tripleo.2005.07.012.

17. Cascaes da Silva F, Valdivia BA, Iop R, Barbosa PJ, da Silva R. Escalas y listas de evaluación de la calidad de estudios científicos. Rev Cuba Inf Cienc Salud. 2013;24(3):295-312.

18. Cömert Kılıç S, Güngörmüş M, Parlak SN. Histologic and histomorphometric assessment of sinus-floor augmentation with beta-tricalcium phosphate alone or in combination with pureplatelet-rich plasma or platelet-rich fibrin: A randomized clinical trial. Clin Implant Dent Relat Res. 2017;19(5)959-67. DOI: $10.1111 /$ cid.12522.

19. Gassling V, Purcz N, Braesen JH, Will M, Gierloff M, Behrens E, et al. Comparison of two different absorbable membranes for the coverage of lateral osteotomy sites in maxillary sinus augmentation: a preliminary study. J Craniomaxillofac Surg. 2013;41(1):76-82. DOI: 10.1016/j.jcms.2012.10.015.

20. Zhang Y, Tangl S, Huber CD, Lin Y, Qiu L, Rausch-Fan X. Effects of Choukroun's platelet-rich fibrin on bone regeneration in combination with deproteinized bovine bone mineral in maxillary sinus augmentation: a histological and histomorphometric study. J Craniomaxillofac Surg. 2012;40(4):321-8. DOI: 10.1016/j.jcms.2011.04.020.

21. Bölükbasi N, Ersanlı S, Keklikoglu N, Basegmez C, Ozdemir T. Sinus augmentation with platelet-rich fibrin in combination with bovine bone graft versus bovine bone graft in combination with collagen membrane. J Oral Implantol. 2015;41(5):58695. DOI: 10.1563/AAID-JOI-D-13-00129.

22. Nizam N, Eren G, Akcalı A, Donos N. Maxillary sinus augmentation with leukocyte and platelet-rich fibrin and deproteinized bovine bone mineral: A split-mouth histological and histomorphometric study. Clin Oral Implants Res. 2018;29(1):67-75. DOI: 10.1111/clr.13044.

23. Boyne PJ, James RA. Grafting of the maxillary sinus floor with autogenous marrow and bone. J Oral Surg. 1980;38(8):613-06.

24. Emmerich D, Att W, Stappert C. Sinus floor elevation using osteotomes: a systematic review and meta-analysis. J Periodontol. 2005;76(8):1237-51. DOI: 10.1902/jop.2005.76.8.1237.

25. Movahedian Attar B, Naghdi N, Etemadi Sh M, Mehdizadeh M. Chin symphysis bone, allograft, and platelet-rich fibrin: is the combination effective in repair of alveolar cleft? J Oral Maxillofac Surg. 2017;75(5):1026-35. DOI: 10.1016/j.joms.2016.12.026.

26. Shawky H, Seifeldin SA. Does platelet-rich fibrin enhance bone quality and quantity of alveolar cleft reconstruction? Cleft Palate Craniofac J. 2016;53(5):597-606. DOI: 10.1597/14-290.

27. Hauser F, Gaydarov N, Badoud I, Vazquez L, Bernard JP, Ammann P. Clinical and histological evaluation of postextraction platelet- 
rich fibrin socket filling: a prospective randomized controlled study. Implant Dent. 2013;22(3):295-303. DOI: 10.1097/ ID.0b013e3182906eb3.

28. Suttapreyasri S, Leepong N. Influence of platelet-rich fibrin on alveolar ridge preservation. J Craniofac Surg. 2013;24(4):108894. DOI: 10.1097/SCS.0b013e31828b6dc3.

29. Kiliç SC, Güngörmüş M. Cone beam computed tomography assessment of maxillary sinus floor augmentation using betatricalcium phosphate alone or in combination with plateletrich plasma: a randomized clinical trial. Int J Oral Maxillofac Implants. 2016;31(6):1367-75. DOI: 10.11607/jomi.5205.

30. Consolo U, Zaffe D, Bertoldi C, Ceccherelli G. Platelet-rich plasma activity on maxillary sinus floor augmentation by autologous bone. Clin Oral Implants Res. 2007;18(2):252-62. DOI: 10.1111/j.1600-0501.2006.01330.x.

31. Kassolis JD, Reynolds MA. Evaluation of the adjunctive benefits of platelet-rich plasma in subantral sinus augmentation. J Craniofac Surg. 2005;16(2):280-7.

32. Raghoebar GM, Schortinghuis J, Liem RS, Ruben JL, van der Wal JE, Vissink A. Does platelet-rich plasma promote remodeling of autologous bone grafts used for augmentation of the maxillary sinus floor? Clin Oral Implants Res. 2005;16(3):349-56. DOI: 10.1111/j.1600-0501.2005.01115.x.

33. Kim BJ, Kwon TK, Baek HS, Hwang DS, Kim CH, Chung IK, et al. A comparative study of the effectiveness of sinus bone grafting with recombinant human bone morphogenetic protein 2-coated tricalcium phosphate and platelet-rich fibrin-mixed tricalcium phosphate in rabbits. Oral Surg Oral Med Oral Pathol Oral Radiol. 2012;113(5):583-92. DOI: 10.1016/j.tripleo.2011.04.029.

34. Jelusic D, Zirk ML, Fienitz T, Plancak D, Puhar I, Rothamel D. Monophasic B-TCP vs. biphasic HA/ß-TCP in two-stage sinus floor augmentation procedures-a prospective randomized clinical trial. Clin Oral Implants Res. 2017;28(10):175-83. DOI: 10.1111/clr.12983.

35. Lezzi G, Degidi M, Piattelli A, Mangano C, Scarano A, Shibli JA, et al. Comparative histological results of different biomaterials used in sinus augmentation procedures: a human study at 6 months. Clin Oral Implants Res. 2012;23(12):1369-76. DOI: 10.1111/j.1600-0501.2011.02308.x.

36. Schulten EA, Prins HJ, Overman JR, Helder MN, ten Bruggenkate CM, Klein-Nulend J. A novel approach revealing the effect of a collagenous membrane on osteoconduction in maxillary sinus floor elevation with $\beta$-tricalcium phosphate. Eur Cell Mater. 2013;25:215-28.

37. Kurkcu M, Benlidayi ME, Cam B, Sertdemir Y. Anorganic bovinederived hydroxyapatite vs $\beta$-tricalcium phosphate in sinus augmentation: a comparative histomorphometric study. J Oral Implantol. 2012;38:519-26. DOI: 10.1563/AAID-JOI-D-11-00061.

38. Thor A, Franke-Stenport V, Johansson CB, Rasmusson L. Early bone formation in human bone grafts treated with platelet-rich plasma: preliminary histomorphometric results. Int J Oral Maxillofac Surg. 2007;36(12):1164-71. DOI: 10.1016/j.ijom.2007.05.023.

39. Marx RE. Platelet-rich plasma: evidence to support its use. J Oral Maxillofac Surg. 2004;62(4):489-96.

40. Diss A, Dohan DM, Mouhyi J, Mahler P. Osteotome sinus floor elevation using Choukroun's platelet-rich fibrin as grafting material: a 1-year prospective pilot study with microthreaded implants. Oral Surg Oral Med Oral Pathol Oral Radiol Endod. 2008;105(5):572-9. DOI: 10.1016/j.tripleo.2007.08.021.

41. Tanaka H, Toyoshima T, Atsuta I, Ayukawa Y, Sasaki M, Matsushita Y, et al. Additional effects of platelet-rich fibrin on bone regeneration in sinus augmentation with deproteinized bovine bone mineral: preliminary results. Implant Dent. 2015;24(6):669-74. DOI: 10.1097/ID.0000000000000306.

42. Ali S, Bakry SA, Abd-Elhakam H. Platelet-rich fibrin in maxillary sinus augmentation: a systematic review. J Oral Implantol. 2015;41(6):746-53. DOI: 10.1563/aaid-joi-D-14-00167.

43. Roldan JC, Jepsen S, Miller J, Freitag S, Rueger DC, Açil Y, et al. Bone formation in the presence of platelet-rich plasma vs. bone morphogenetic protein-7. Bone. 2004;34(1):80-90. DOI: 10.1016/j.bone.2003.09.011.

44. Bowers GM, Chadroff B, Carnevale R, Mellonig J, Corio R, Emerson J, et al. Histologic evaluation of new attachment apparatus formation in humans. Part III. J Periodontol. 1989;60(12):683-93. DOI: 10.1902/jop.1989.60.12.683.

45. Wei L, Miron RJ, Shi B, Zhang Y. Osteoinductive and osteopromotive variability among different demineralized bone allografts. Clin Implant Dent Relat Res. 2015;17(3):533-42. DOI: 10.1111/cid.12118.

46. Schwartz Z, Somers A, Mellonig JT, Carnes DL Jr, Dean DD, Cochran DL, et al. Ability of commercial demineralized freezedried bone allograft to induce new bone formation is dependent on donor age but not gender. J Periodontol. 1998;69(4):470-8.

47. Yang YI, Seol DL, Kim HI, Cho MH, Lee SJ. Composite fibrin and collagen scaffold to enhance tissue regeneration and angiogenesis. Curr Appl Phys. 2007;7(1):103-7.

48. Browaeys H, Bouvry P, De Bruyn H. A literature review on biomaterials in sinus augmentation procedures. Clin Implant Dent Relat Res. 2007;9(3):166-77. DOI: 10.1111/j.1708-8208.2007.00050.x.

49. Tadjoedin ES, de Lange GL, Bronckers AL, Lyaruu DM, Burger EH. Deproteinized cancellous bovine bone (Bio-Oss) as bone substitute for sinus floor elevation. A retrospective, histomorphometrical study of five cases. J Clin Periodontol. 2003;30(3):261-70. DOI: 10.1034/j.1600-051x.2003.01099.x.

50. Piattelli M, Favero GA., Scarano A, Orsini G, Piattelli A. Bone reactions to anorganic bovine bone (Bio-Oss) used in sinus augmentation procedures: a histologic long-term report of 20 cases in humans. Int J Oral Maxillofac Implants. 1999;14(6):835-40.

51. Hallman M, Lundgren S, Sennerby L. Histologic analysis of clinical biopsies taken 6 months and 3 years after maxillary sinus floor augmentation with $80 \%$ bovine hydroxyapatite and $20 \%$ autogenous bone mixed with fibrin glue. Clin Implant Dent Relat Res. 2001;3(2):87-96.

52. Yildirim M, Spiekermann H, Biesterfeld S, Edelhoff D. Maxillary sinus augmentation using xenogenic bone substitute material Bio-Oss in combination with venous blood. A histologic and histomorphometric study in humans. Clin Oral Implants Res. 2000;11(3):217-29.

53. Orsini G, Traini T, Scarano A, Degidi M, Perrotti V, Piccirilli M, et al. Maxillary sinus augmentation with Bio-Oss particles: a light, scanning, and transmission electron microscopy study in man. J Biomedl Mater Res B Appl Biomater. 2005;74(1):448-57.

54. Degidi M, Artese L, Rubini C, Perrotti V, Iezzi G, Piattelli A. Microvessel density and vascular endothelial growth factor expression in sinus augmentation using Bio-Oss. Oral Dis. 2006;12(5):469-75. DOI: 10.1111/j.1601-0825.2006.01222.x.

55. Lee YM, Shin SY, Kim JY, Kye SB, Ku Y, Rhyu IC. Bone reaction to bovine hydroxyapatite for maxillary sinus floor augmentation: histologic results in humans. Int J Periodontics Restorative Dent. 2006;26(5):471-81.

56. Sartori S, Silvestri M, Forni F, Icaro Cornaglia A, Tesei P, Cattaneo V. Ten-year follow-up in a maxillary sinus augmentation using anorganic bovine bone (Bio-Oss). A case report with histomorphometric evaluation. Clin Oral Implants Res. 2003;14(3):369-72.

57. Traini T, Valentini P, Lezzi G, Piattelli A. A histologic and histomorphometric evaluation of anorganic bovine bone retrieved 9 years after a sinus augmentation procedure. J Periodontol. 2007;78(5):955-61. DOI: 10.1902/jop.2007.060308.

58. Miron RJ, Zucchelli G, Pikos MA, Salama M, Lee S, Guillemette $\mathrm{V}$, et al. Use of platelet-rich fibrin in regenerative dentistry: a systematic review. Clin Oral Investig. 2017;21(6):1913-27. DOI: 10.1007/s00784-017-2133-z.

59. Agarwal A, Gupta ND, Jain A. Platelet rich fibrin combined with decalcified freeze-dried bone allograft for the treatment of human intrabony periodontal defects: a randomized split mouth clinical trail. Acta Odontol Scand. 2016;74(1):36-43. DOI: 10.3109/00016357.2015.1035672.

60. Toffler M, Toscano N, Holtzclaw D. Osteotome-mediated sinus floor elevation using only platelet-rich fibrin: an early report 
on 110 patients. Implant Dent. 2010;19(5):447-56. DOI: 10.1097/ ID.0b013e3181f57288.

61. Vijayalakshmi R, Rajmohan CS, Deepalakshmi D, Sivakami G. Use of platelet rich fibrin in a fenestration defect around an implant. J Indian Soc Periodontol. 2012;16(1):108-12. DOI: 10.4103/0972$124 X .94616$.

62. Kawase T, Kamiya M, Kobayashi M, Tanaka T, Okuda K, Wolff LF, et al. The heat-compression technique for the conversion of platelet-rich fibrin preparation to a barrier membrane with a reduced rate of biodegradation. J Biomed Mater Res B Appl Biomater. 2015;103(4):825-31. DOI: 10.1002/jbm.b.33262.

63. Infante-Cossío P, Gutiérrez-Pérez JL, Torres-Lagares D, GarcíaPerla García A, González-Padilla JD. Relleno de cavidades óseas en cirugía maxilofacial con materiales autólogos. Rev Esp Cirug Oral y Maxilofac. 2007;29(1):7-19.

64. Kalaaji A, Lilja J, Elander A, Friede H. Tibia as donor site for alveolar bone grafting in patients with cleft lip and palate: long-term experience. Scand J Plast Reconstr Surg Hand Surg. 2001;35(1):35-42.

65. Horswell BB, Henderson JM. Secondary osteoplasty of the alveolar cleft defect. J Oral Maxillofac Surg. 2003;61(9):1082-90. DOI: 10.1016/s0278-2391(03)00322-7.

66. Eichhorn W, Blessmann M, Pohlenz P, Blake FA, Gehrke G, Schmelzle R, et al. Primary osteoplasty using calvarian bone in patients with cleft lip, alveolus and palate. J Craniomaxillofac Surg. 2009;37(8):429-33. DOI: 10.1016/j.jcms.2009.07.009.

67. Johnson K. A study of the dimensional changes occurring in the maxilla following tooth extraction. Aust Dent J. 1969;14(4):241-4. DOI: 10.1111/j.1834-7819.1969.tb06001.x.

68. Pietrokovski J, Massler M. Alveolar ridge resorption following tooth extraction. J Prosthet Dent. 1967;17(1):21-7. DOI: 10.1016/00223913(67)90046-7.
69. Lekovic V, Kenney EB, Weinlaender M, Han T, Klokkevold P, Nedic $\mathrm{M}$, et al. A bone regenerative approach to alveolar ridge maintenance following tooth extraction. Report of 10 cases. J Periodontol. 1997;68(6):563-70. DOI: 10.1902/jop.1997.68.6.563.

70. Carlsson GE, Bergman B, Hedegard B. Changes in contour of the maxillary alveolar process under immediate dentures. A longitudinal clinical and x-ray cephalometric study covering 5 years. Acta Odontol Scand. 1967;25(1):45-75. DOI: 10.3109/00016356709072522.

71. Schneider R. Prosthetic concerns about atrophic alveolar ridges. Postgrad Dent. 1999;6(2):3-7.

72. Denissen HW, Kalk W, Veldhuis HA, van Waas MA. Anatomic consideration for preventive implantation. Int J Oral Maxillofac Implants. 1993;8(2):191-6.

73. Araujo MG, Lindhe J. Dimensional ridge alterations following tooth extraction. An experimental study in the dog. J Clin Periodontol. 2005;32(2):212-8.

74. Brkovic BM, Prasad HS, Konandreas G, Milan R, Antunovic D, Sándor GK, et al. Simple preservation of a maxillary extraction socket using beta-tricalcium phosphate with type I collagen: preliminary clinical and histomorphometric observations. J Can Dent Assoc. 2008;74(6):523-8.

75. Lang NP, Pun L, Lau KY, Li KY, Wong MC. A systematic review on survival and success rates of implants placed immediately into fresh extraction sockets after at least 1 year. Clin Oral Implants Res. 2012;23(5):39-66. DOI: 10.1111/j.1600-0501.2011.02372.x.

76. He L, Lin Y, Hu X, Zhang Y, Wu H. A comparative study of platelet-rich fibrin (PRF) and platelet-rich plasma (PRP) on the effect of proliferation and differentiation of rat osteoblasts in vitro. Oral Surg Oral Med Oral Pathol Oral Radiol Endod. 2009;108(5):707-13. DOI: 10.1016/j.tripleo.2009.06.044. 